\title{
LA OPINIÓN DE LA CIUDADANÍA COMO DATO VALIOSO PARA LA ACERTADA TOMA DE DECISIONES TRIBUTARIAS: ¿USO PROSPECTIVO DE BARÓMETROS FISCALES EN TIEMPOS DE REFORMA?
}

\author{
María Amparo Grau Ruiz \\ Catedrática de Derecho Financiero y Tributario \\ Universidad Complutense de Madrid (España) \\ Directora de la Revista Técnica Tributaria
}

Las normas, cuanto más ajustadas a la realidad social, son (y también se perciben como) más justas. En la situación actual, por la dureza de las circunstancias y los medios con los que se cuenta, es fácilmente comprensible que las demandas y las expectativas de la ciudadanía en lo tocante a los sistemas fiscales y su buena administración sean crecientes. Además, la aspiración a la justicia tributaria ha de acompañarse de una mayor eficacia y eficiencia en el gasto público -ya que materialmente ha de legitimar las nuevas exigencias-, en especial tras haber entrado en la última Década de acción para el logro de los objetivos de la Agenda 2030 de la Organización de las Naciones Unidas, que orienta no sólo la política fiscal europea sino también la mundial.

Indiscutiblemente, el fomento de la participación crítica es deseable en todo proceso de mejora continua. Con ella se puede ampliar la perspectiva del conocimiento de la realidad cotidiana vivida en cada uno de los sectores de la maltrecha economía. Por ese motivo, al margen de que puedan arbitrarse novedosos mecanismos (extensivos e intensivos) de recogida de datos objetivos de los obligados tributarios (sobre sus actividades, operaciones, movimientos, etcétera) a través de procedimientos casi totalmente automatizados, es siempre oportuno recabar también el parecer de la sociedad sobre el Derecho financiero $\mathrm{y}$ tributario vigente y tomar el pulso a su aplicación a partir de datos subjetivos. Incluso cabe plantear que se realicen estos análisis de manera prospectiva, sondeando algunas alternativas consideradas en el marco de posibles líneas de reforma para promover la recuperación y remediar 
los efectos de la actual crisis en todas sus dimensiones (sanitaria, económica y climática). Desde luego, no parece razonable pasar por alto cómo afecta todo este cúmulo de condicionantes a la evolución de la moral fiscal de los españoles ${ }^{1}$.

En este sentido, conviene llamar aquí la atención sobre el posible uso de barómetros y, en su caso, su configuración interdisciplinar para responder mejor a los ejes transversales (la transición ecológica, la transformación digital, la igualdad de género y la cohesión social y territorial) fijados en el Plan de Recuperación, Transformación y Resiliencia recientemente elaborado por el Gobierno de España, que, como es sabido, incluye la «modernización del sistema fiscal para un crecimiento inclusivo y sostenible» como política palanca de reforma estructural ${ }^{2}$.

Por una parte, las herramientas para conocer el estado de la opinión pública facilitan la toma de decisiones normativas debidamente informadas. Así, la utilización de barómetros es sobradamente conocida en diversas áreas (por ejemplo, el Parlamento Europeo periódicamente tantea las preferencias de los ciudadanos de la Unión sobre ciertos temas ${ }^{3}$ ). Conviene resaltar que, con frecuencia, su propósito es contribuir a garantizar la aceptación de las normas que atraviesan la fase de creación o son susceptibles de modificación. En última instancia, esta forma de actuar coadyuva a la expansión del cumplimiento voluntario, por haberse reforzado la legitimidad de la actuación legislativa.

Por otra parte, estratégicamente, el actual puede ser un buen momento para valorar, en su conjunto, la redefinición de la obtención y explotación de datos de los distintos grupos y agentes sociales sometidos a imposición y de quienes se benefician de algún modo de financiación pública. Adicionalmente, si la paulatina recopilación masiva de información dejase de limitarse de forma casi exclusiva a datos meramente objetivos y se extendiera a los de naturaleza subjetiva con el fin de sopesar la visión de los receptores de las normas sobre los modos más eficientes de alcanzar fines comunes, se estarían fomentando las alianzas público-privadas conforme propone el Objetivo de Desarrollo Sostenible número 17 de la Agenda 2030. Esta tarea bien podría servir para reforzar la confianza en las instituciones públicas.

De hecho, ya hay iniciativas privadas que se ocupan de analizar a

1 López Laborda, J.; Sanz Arcega, E., La moral fiscal de los españoles, reexaminada, Revista de economía aplicada, Vol. 24, No. 70, 2016, pp. 53-76. Capa, C., Barómetro fiscal: ¿pagamos lo que debemos?, Escritura pública, No. 98, 2016, pp. 48-51. Sanz Arcega, E., Tres ensayos de sociología tributaria con microdatos: redistribución, principio de beneficio y moral fiscal, Tesis doctoral dirigida por julio López Laborda, Universidad de Zaragoza, 2014. López Laborda, J.; Sanz Arcega, E., La moral fiscal de los españoles, revisitada, Documentos de Trabajo FUNCAS, No. 722, 2013.

2 Es ésta una de las diez políticas tractoras que van a incidir directamente en aquellos sectores productivos con mayor capacidad de transformación de nuestro tejido económico y social.https:// www.lamoncloa.gob.es/temas/fondos-recuperacion/Paginas/plan-de-recuperacion.aspx [última consulta 10 de junio de 2021].

3 Estos pueden consultarse en https://europa.eu/eurobarometer/surveys/browse/all;search= [última consulta 10 de junio de 2021]. 
través de barómetros aspectos específicos de la tributación en distintas partes del mundo. A menudo resultan de utilidad para la adecuada gestión empresarial ${ }^{4}$. En Derecho comparado, por ejemplo, en Estados Unidos, el Tax Council y Ernst \& Young LLP elaboran periódicamente el Barómetro de la Política Fiscal de las Empresas, que proporciona información sobre las percepciones de la comunidad empresarial sobre cuestiones clave de la política fiscal de las empresas (a escala federal y mundial).

En nuestro país, tradicionalmente, el área de sociología tributaria del Instituto de Estudios Fiscales ha realizado numerosos estudios sobre las opiniones y actitudes fiscales de los españoles desde 1995 cada año ${ }^{5}$, por lo que su dilatada experiencia podría tomarse como referencia.

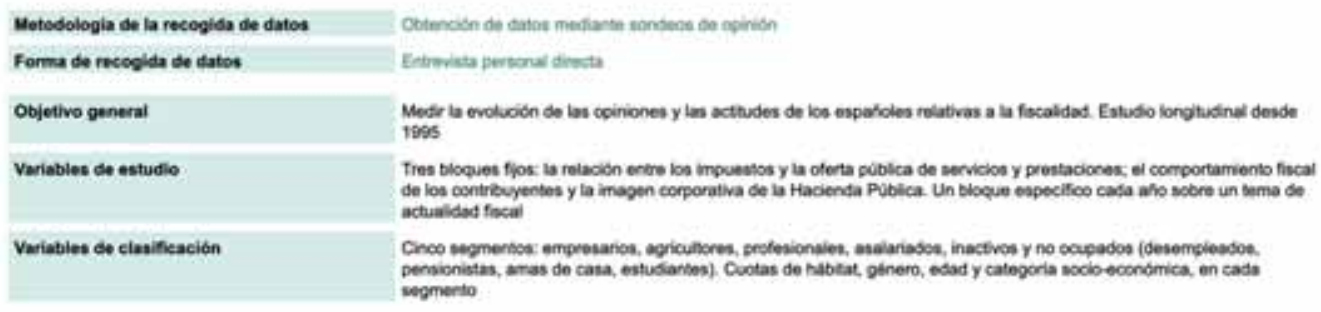

Fuente: $I N E^{6}$

4 EY, Business Tax Policy Barometer 2021 (US), disponible en https://www.ey.com/en_us/tax/ business-tax-policy-barometer KPMG, European Family Business Barometer 2019. KPMG Meijburg \& Co disponible en https://meijburg.com/overigen/european-family-business-barometer. KPMG, Tax in the Boardroom, Effective governance - KPMG Tax Barometer (NZ), disponible en https://assets.kpmg/ content/dam/kpmg/nz/pdf/2020/08/tax-barometer-2020.pdf [última consulta 10 de junio de 2021]. 5 IEF - Area de Sociología Tributaria, Ciudadanos, contribuyentes y expertos: opiniones y actitudes fiscales de los españoles en 1997 [Barómetro fiscal], Instituto de Estudios Fiscales, Madrid, 1998; San Vicente, M.; Delgado Lobo, M.L., Evolución de la opinión fiscal a través de datos de encuesta, Papeles de trabajo del Instituto de Estudios Fiscales. Serie economía, No. 9, 1998, pp. 1-28. Sanz Sanz, J.F.; Salinas Jiménez, J.; Delgado Lobo, M.L., La opinión fiscal a través del barómetro del Instituto de Estudios Fiscales (1995-2000), Economistas, Vol. 19, No. 88, 2001, pp. 81-93. Delgado Lobo, M.L., Opiniones y actitudes fiscales de los españoles en 2004 [Barómetro fiscal], Instituto de Estudios Fiscales, Madrid, 2005. Goenaga Ruiz de Zuazu, M.; Pérez López, C., El barómetro fiscal del Instituto de Estudios Fiscales: una herramienta para la medición de la opinión fiscal, Revista de Estadística y Sociedad, No. 45, 2011, pp. 28-30. Cerno, L.; Pérez López, C.; Sanz Arcega, E., Determinantes de la satisfacción de los españoles con las prestaciones y servicios públicos: un enfoque de sociología tributaria con microdatos, Hacienda Pública Española, No. 220, 2017, pp. 57-87. 6 https://ine.es/dyngs/IOE/es/operacion.htm?id=1259931153317 [última consulta 10 de junio de 2021]. 
Los contenidos de los tres capítulos iniciales suelen repertirse, como se detallan a continuación:

CAPÍTULO 1. RELACIÓN ENTRE LOS IMPUESTOS Y LA OFERTA PÚBLICA DE SERVICIOS Y PRESTACIONES

1.1. Utilización de servicios públicos y prestaciones sociales en el último año

1.2. Índice de satisfacción de los usuarios

1.3. Evaluación general de la oferta pública de servicios y prestaciones

1.4. Evaluación de diferentes servicios públicos y prestaciones sociales

CAPÍTULO 2. EL COMPORTAMIENTO DE LOS CONTRIBUYENTES

2.1. Evolución del grado de cumplimiento fiscal fiscal

2.2. Opiniones sobre el fraude

CAPÍTULO 3. IMAGEN CORPORATIVA DE LA HACIENDA PÚBLICA

3.1. La cumplimentación de las declaraciones tributarias

3.2. Los servicios al contribuyente de la Agencia Tributaria

3.3. Valoración de la Hacienda Pública como institución
Los contenidos del último capítulo varían año tras año, en función de las prioridades que se estimen oportunas. Pueden comprobarse los temas trabajados en los últimos tres años:

En 2017 en el CAPÍTULO 4 se analizó EL PAGO DE IMPUESTOS POR LAS GRANDES EMPRESAS $^{7}$

4.1. Percepción del pago de impuestos por las grandes empresas

4.2. Motivos por los que las grandes empresas pagan menos impuestos y grado de justificación

4.3. Propuesta más adecuada para conseguir que las grandes empresas paguen más impuestos en el futuro

En 2018 el CAPÍTULO 4 cubrió LA INTEGRACIÓN EUROPEA EN MATERIA DE INGRESOS Y GASTOS PÚBLICOS $^{8}$

4.1. Conformidad con las propuestas debatidas en la Unión Europea

4.2. Posibilidad de fijar un sistema de garantía de depósito común a nivel europeo

4.3. Valoración de la creación de un Misterio de Economía y Hacienda europeo

\subsection{Opinión sobre la} homogeneización de la regulación de impuestos

7 Opiniones y actitudes fiscales de los españoles en 2017, DOCUMENTOS DE TRABAJO del IEF, No. 13/2018. Disponible en https://www.ief.es/docs/destacados/publicaciones/documentos_trabajo/ 2018_13.pdf [última consulta 10 de junio de 2021].

8 DIRECCIÓN DE ESTUDIOS: Opiniones y actitudes fiscales de los españoles en 2018, DOCUMENTOS DE TRABAJO del IEF, No. 16, 2019. Disponible en https://www.ief.es/docs/destacados/ publicaciones/documentos_trabajo/2019_16.pdf [última consulta 10 de junio de 2021]. 
En 2019 el CAPÍTULO 4 se centró en LA IMPOSICIÓN SOBRE LA RIQUEZA $^{9}$

4.1. Conformidad con los impuestos que gravan la riqueza

4.2. Mínimo exento a partir del cual se debería tributar por el Impuesto sobre el Patrimonio

4.3. Impuesto sobre Sucesiones y Donaciones en función del grado de parentesco

4.4. Opinión sobre la armonización de la regulación del Impuesto sobre Sucesiones y Donaciones en territorio español

Ante la magnitud y complejidad de los cambios a afrontar en el momento presente, desde una perspectiva jurídica, quizás podrían emplearse con mayor frecuencia barómetros fiscales para procurar una mejor técnica legislativa y hacer progresar la buena administración. Sobre todo, cuando es previsible la pronta reinvención de algunos tributos o incluso la aparición de nuevos, o el surgimiento de modalidades de gestión más «inteligentes» de todos ellos a la vista de los avances tecnológicos.

Es obvio que lo practicable acaba incidiendo inevitablemente en el diseño normativo. A medida que se abren nuevas vías cuasi ilimitadas para que la Administración tributaria pueda recabar información, cobra pleno sentido la adopción de normas que perfectamente pueden ajustarse en tiempo real. Así, se hace posible la toma de decisiones a partir de datos estrechamente conectados a la realidad social del tiempo en que dichas normas han de ser aplicadas.

Ahora bien, nadie puede conocer mejor que el propio sujeto inmerso en la vida práctica del sector de que se trate (y los que con él interactúan) cómo afecta o puede afectar la regulación vigente y sus posibles cambios al desarrollo de su actividad, las limitaciones que se encuentran y los modos de superarlas.

Uno de los factores determinantes del éxito de cualquier reforma legislativa es la percepción social de un orden fiscal justo que se adapta a las necesidades de los tiempos. La «apropiación» por la ciudadanía de los objetivos perseguidos resulta fundamental para evitar cualquier fracaso ulterior. Por esa razón, interesa potenciar el uso de los instrumentos disponibles para anticipar cualquier tipo de problemas reales que puedan quedar soslayados, tratando de conocer con antelación las reacciones de la opinión pública ante diferentes opciones propuestas.

Al mismo tiempo, mediante el uso de barómetros fiscales también puede hacerse patente a la sociedad el importante rol que juegan los sistemas fiscales en la transformación económica, insistiendo en la buena gobernanza fiscal, conforme a los principios de justicia tributaria y los fines constitucionales de política económica y social.

Por último, no debe olvidarse que la Hacienda pública moderna tiene suficientes medios a su alcance para hacer un mejor uso de las técnicas de estímulo previstas en el ordenamiento

9 DIRECCIÓN DE ESTUDIOS: Opiniones y actitudes fiscales de los españoles en 2019, DOCUMENTOS DE TRABAJO del IEF, No. 9, 2020. Disponible en https://www.ief.es/docs/destacados/ publicaciones/documentos_trabajo/2020_09.pdf [última consulta 10 de junio de 2021]. 
jurídico-financiero, contando con la colaboración del sector privado en la satisfacción de fines públicos. Por ejemplo, el uso eficaz de barómetros puede ser esperanzador para detectar fallos en la aplicación del presupuesto de gastos fiscales y diseñar fórmulas de control útiles cuando se adoptan normas multipropósito (pues éstas parecen proliferar y es relativamente difícil medir su verdadera eficacia).

Asimismo, convendría definir las sinergias que pueden ser fruto de una actuación coordinada a la hora de enlazar barómetros en distintos ámbitos (territoriales o materiales); o la relación con las estadísticas disponibles en virtud de acuerdos de cooperación institucional entre diversas autoridades; o con asociaciones profesionales de especialistas.

En definitiva, los esfuerzos en la construcción de un marco normativo más preciso, ajustado a la realidad y que aumente la seguridad jurídica, pueden conducir a una administración confiable ${ }^{10}$. Ésta debe esforzarse por mejorar los canales de comunicación con la comunidad, explicando las políticas fiscales de manera compresible en un ejercicio de transparencia y estar abierta a recibir retroalimentación de manera dinámica. Esto es trascendental, justo en la coyuntura que atravesamos, cuando se observa una cierta tensión en la visión de conjunto del sistema tributario, por debatirse más o menos veladamente el mayor alcance que quiere conferirse al principio de beneficio frente al de capacidad económica.

No se trata de esforzarse por adivinar futuribles, sino de encontrar formas sensatas de orientar bien los pasos a dar en el presente hacia el futuro deseado, compartiendo opiniones y promoviendo la escucha activa a los contribuyentes, demostrando que se les entiende. Quizás mediante el uso de barómetros podría prestárseles más atención en la búsqueda de respuestas para compaginar de manera equilibrada justicia y competitividad, promoviendo el empleo y la productividad. Parece un contrasentido que cuando mayor es la necesidad de acercamiento para revivir la actividad económica, algunas autoridades tributarias tengan la tentación de optar por soluciones fáciles alejándose de la realidad (con bases sustitutivas o tipos mínimos).

10 Cerno, L., Medición de la percepción social sobre el funcionamiento de la administración tributaria en España, Estadística española, Vol. 58, No. 189, 2016, pp. 81-94. 